\title{
Cooperative mechanism for anchoring highly polar molecules at an ionic surface
}

\author{
J. Schütte, ${ }^{*}$ R. Bechstein, ${ }^{\dagger}$ M. Rohlfing, and M. Reichling \\ Fachbereich Physik, Universität Osnabrück, Barbarastraße 7, 49076 Osnabrück, Germany \\ A. Kühnle \\ Institut für Physikalische Chemie, Johannes Gutenberg-Universität Mainz, Jakob-Welder-Weg 11, 55099 Mainz, Germany
}

(Received 22 October 2009; published 19 November 2009)

\begin{abstract}
Structure formation of the highly polar molecule cytosine on the (111) cleavage plane of calcium fluoride is investigated in ultrahigh vacuum using noncontact atomic force microscopy at room temperature. Molecules form well-defined trimer structures, covering the surface as homogeneously distributed stable structures. Density-functional theory calculations yield a diffusion barrier of about $0.5 \mathrm{eV}$ for individual molecules suggesting that they are mobile at room temperature. Furthermore, it is predicted that the molecules can form trimers in a configuration allowing all molecules to attain their optimum adsorption position on the substrate. As the trimer geometry facilitates hydrogen bonding between the molecules within the trimer, we conclude that the stabilization of individual diffusing molecules into stable trimers is due to a cooperative mechanism involving polar interactions between molecules and substrate as well as hydrogen bonding between molecules.
\end{abstract}

DOI: $10.1103 /$ PhysRevB.80.205421

PACS number(s): 68.43.Fg, 68.37.Ps, 81.16.Dn

Precise control of molecular structure formation on surfaces is most important for creating functional molecular devices for future molecular (opto) electronics applications. ${ }^{1}$ Molecular self-assembly has been identified as a powerful method to create tailor-made structures ranging form clusters, unidirectional rows to complex molecular networks. ${ }^{2-5}$ Until now, the vast majority of experimental molecular adsorption studies has been carried out on metallic or semiconducting rather than insulating substrates. ${ }^{6,7}$ This is largely due to the fact that many surface-science techniques require conductive substrates and are, therefore, not suited for studying insulating materials. In particular, scanning tunneling microscopy cannot be applied for imaging of insulating surfaces. Only very recently, direct-space imaging of molecular self-assembly has been extended to dielectric substrates using noncontact atomic force microscopy (NC-AFM) ${ }^{8-21}$ So far, however, controlled structure formation has been hindered by weak and unspecific molecule-substrate interactions, frequently leading to clustering and bulk crystal formation of the molecules at step edges ${ }^{15}$ or aggregation into structures of several nanometers in width. ${ }^{16,17}$ Recently, cleavage step edges and patterning by electron irradiation on $\operatorname{KBr}(001)$ (Ref. 22) have been exploited as strategies to restrict molecular motion on insulating surfaces and to create stable molecular structures. ${ }^{18-21}$

Here, we report upon a rational approach toward molecular structure formation based on a careful selection of a system having well-balanced molecule-molecule as well as molecule-substrate interactions. Molecular trimers are anchored at an ionic surface by employing the electrostatic interaction between the molecules having a high dipole moment and an ionic crystal, namely, $\mathrm{CaF}_{2}(111)$. The molecule of choice is cytosine, $\mathrm{C}_{4} \mathrm{H}_{5} \mathrm{~N}_{3} \mathrm{O}$, as it (i) provides a high dipole moment $[\sim 6$ to $8 \mathrm{D}$ (Refs. $23-27)]$ with (ii) an estimated distance between the partial charges of $\approx 4 \AA$ close to atomic distances of the $\mathrm{CaF}_{2}(111)$ surface. Moreover, the molecular structure allows for formation of intermolecular hydrogen bonds, ${ }^{28}$ enabling the stabilization of molecular trimers at room temperature. From an interplay between ex- periment and density-functional theory (DFT) calculations we infer the details of trimer formation. In its optimum adsorption position, an individual molecule anchors with the negatively charged part above a calcium ion in the second layer while the positively charged part of the molecule is close to two fluorine ions in the first layer. The calculated diffusion energy of $0.5 \mathrm{eV}$ (with an uncertainty of $\gtrsim 0.1 \mathrm{eV}$ ) indicates that individual molecules should be mobile at room temperature, in good agreement with the evidence from experiment. When arranged in a hydrogen-bonded trimer structure, however, the diffusion barrier can be anticipated as three times as high as the diffusion barrier for an individual molecule, which cannot be overcome at room temperature.

All measurements were performed in ultrahigh vacuum (UHV) at room temperature using NC-AFM operated in the frequency modulation mode. ${ }^{29}$ The experimental setup has been described previously. ${ }^{30}$ In NC-AFM, the change in eigenfrequency, $\Delta f$, of an oscillating cantilever is the imaging signal that is fed into a distance feedback control loop. For all images shown here, the distance feedback loop was set very slow in order to provide quasiconstant-height images while still following the overall tilt of the sample surface. ${ }^{31,32}$ Fast and slow scan directions are indicated by the arrows in the upper right corner in the images. The images shown here are selected from a large number of measurement sessions taken with different tips and after repeated sample preparation. Multiple-tip artifacts appeared to occur rather frequently for the presented system.

The $\mathrm{CaF}_{2}$ crystals were purchased from Korth Kristalle (Altenholz, Germany). After insertion into UHV, the crystals were outgassed at $425 \mathrm{~K}$ for $2 \mathrm{~h}$ before cleavage. Our cleavage procedure results in atomically flat terraces of about $1 \mu \mathrm{m}^{2}$ dimensions. ${ }^{33}$ After cleavage, the samples were annealed at $350 \mathrm{~K}$ for $1 \mathrm{~h}$ to remove surface charges. ${ }^{34}$ The cleanliness of the surface and the performance of the tip were carefully checked before deposition of the molecules. On bare substrate surfaces atomic resolution was always achieved. Cleaved $\mathrm{CaF}_{2}(111)$ has the stacking sequence $\mathrm{F}^{-}-\mathrm{Ca}^{2+}-\mathrm{F}^{-}$and is fluorine terminated as shown in the model 

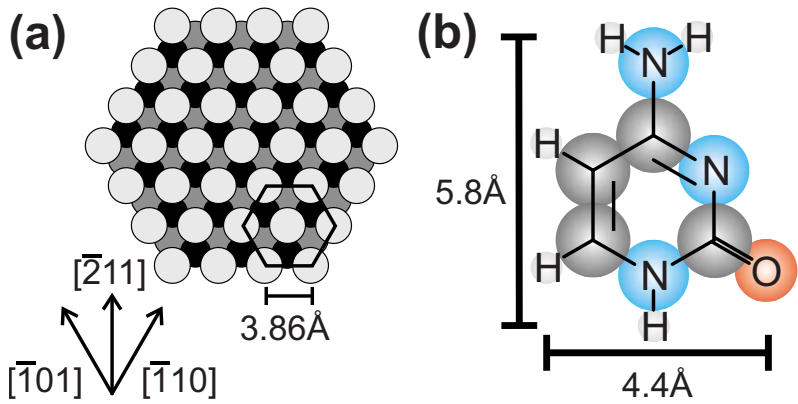

FIG. 1. (Color online) Model of surface and molecule used in this study. (a) Sketch of the $\mathrm{CaF}_{2}(111)$ surface. Bright and dark gray circles represent fluorine ions of the first and third layer, respectively. In between, $\mathrm{Ca}^{2+}$ ions are located (black circles). (b) Sketch of the cytosine molecule. Circles mark carbon atoms, nitrogen atoms $(\mathrm{N})$, hydrogen atoms $(\mathrm{H})$ or an oxygen atom $(\mathrm{O})$.

in Fig. 1(a). NC-AFM imaging allows for unambiguous identification of the fluorine and calcium sublattices. ${ }^{35-37}$ The surface has a threefold symmetry and the nearest-neighbor distance between two fluorine atoms is $3.86 \AA$. A model of cytosine is shown in Fig. 1(b). The molecules (SigmaAldrich, Steinheim, Germany) were thoroughly outgassed at $360 \mathrm{~K}$ for $24 \mathrm{~h}$ before sublimation onto the surface from a homemade Knudsen cell heated to $400 \mathrm{~K}$. The coverage shown in this study was obtained after $40 \mathrm{~s}$ deposition onto the sample held at room temperature positioned $\sim 9 \mathrm{~cm}$ apart from the Knudsen cell.

An overview over the molecule-covered surface is shown in Fig. 2. The total coverage amounts to $(7 \pm 1) \%$ of an entirely covered surface. Molecular features are observed to be homogeneously distributed over the surface. The size of

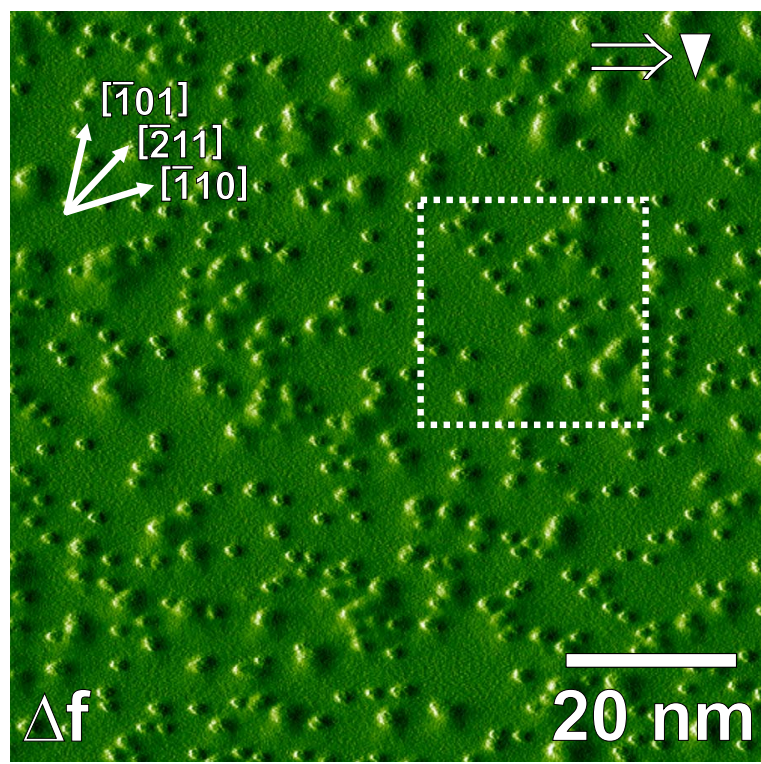

FIG. 2. (Color online) Overview of $\mathrm{a} \mathrm{CaF}_{2}(111)$ terrace covered with cytosine molecules. Homogeneously distributed clusters are observed. The two most dominant structures accounting for $(75 \pm 3) \%$ of all clusters have well-defined size and appearance. The marked area is reproduced in high resolution in Fig. 3. Average $\Delta f=-7.7 \mathrm{~Hz}$.
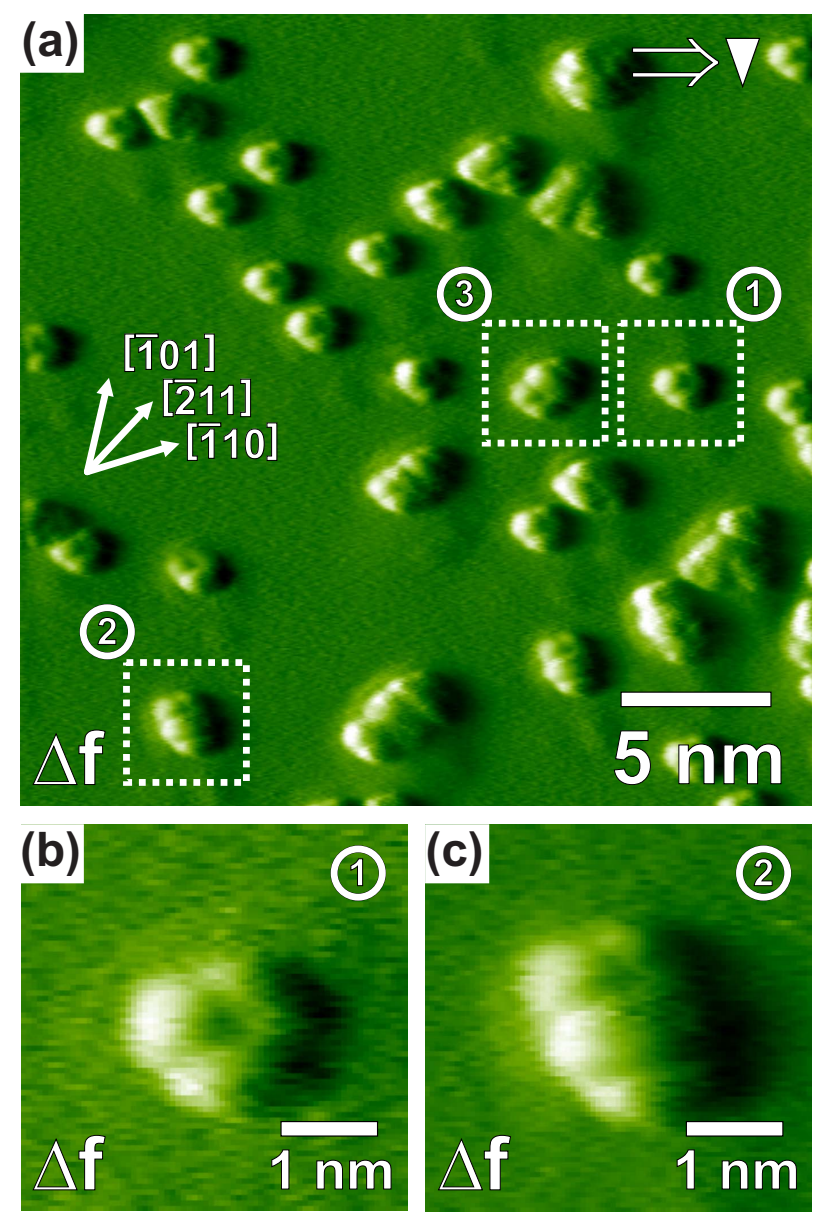

FIG. 3. (Color online) (a) High-resolution image of the area marked in Fig. 2. Besides the dominant molecular trimer structure as marked by box (1), also tetramers [boxes (2) and (3)] and a few less well-defined structures are observed. The trimer structures are by far the most frequently observed clusters. (b) Zoom into the trimer structure marked with (1) in (a). (c) Zoom into the tetramer structure marked with (2) in (a). Average $\Delta f=-6.2 \mathrm{~Hz}$. Note that all images shown here are taken from left to right. Due to the slow feedback loop setting used in this study, the protruding molecular features appear bright on the left side and have a dark "shadow" to the right.

these features is significantly larger than what is expected for single molecules. Thus, we conclude that individual molecules diffuse on the surface and nucleate into clusters. Most of these clusters have a well-defined size and appearance while only a few larger and structurally less regularly shaped clusters are observed. The underlying $\mathrm{CaF}_{2}(111)$ lattice cannot be simultaneously resolved together with the molecules, for similar reasons as those explained previously for formate on $\mathrm{MgO}(100) .{ }^{38}$ We can, however, unambiguously assign the crystal directions by comparison to atomically resolved images of bare $\mathrm{CaF}_{2}(111)$ taken before molecule deposition.

A more detailed view of the area marked in Fig. 2 is shown in Fig. 3(a). In this image, the most dominant, welldefined structures and several exceptional clusters are clearly visible. One of these dominant clusters [marked by box(1)] is reproduced with higher resolution in Fig. 3(b). Each cluster is composed of three lobes and we conclude that each of 
(a)

(c)
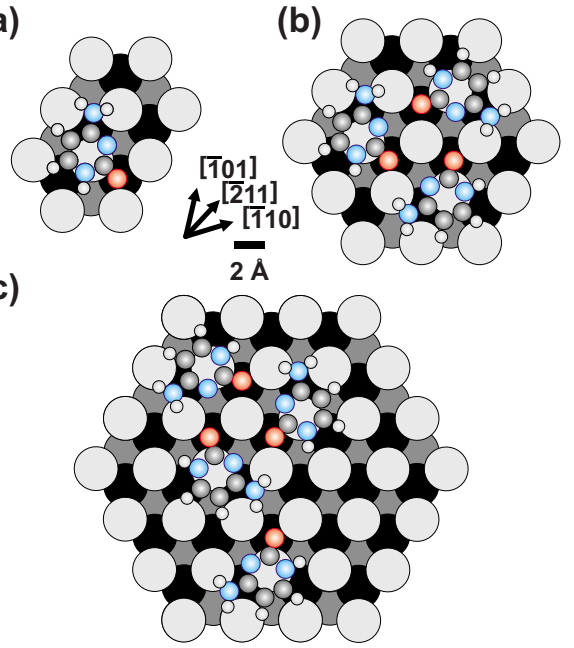

FIG. 4. (Color online) (a) Optimum adsorption position as obtained by DFT calculations. (b) Model of the trimer structure based on (a). Three molecules forming a hydrogen-bonded ring are positioned onto the surface with all molecules adopting the optimum adsorption position shown in (a). (c) Model of the tetramer structure based on (b) and an additional molecule adopting the optimum adsorption position as shown in (a).

them represents a cytosine molecule. Note that the clusters do not exhibit a sixfold but only threefold symmetry due to the underlying substrate. Besides these trimers, slightly larger clusters are observed [boxes (2) and (3)], see also Fig. $3(\mathrm{c})$. These structures resemble the trimers with one additional feature attached to the trimer. Due to the threefold lattice symmetry, these tetramers appear in three different orientations, two of which are shown in the boxes (2) and (3). By far the most of the agglomerates found on the surface can be associated to trimer $(58 \pm 3) \%$ and tetramer clusters $(17 \pm 3) \%$. A small fraction of other species exist on the surface, which cannot be assigned unambiguously. These features might originate from impurities such as adsorbed molecules from the residual gas, especially water, or defects on the surface.

DFT calculations were performed to unravel the optimum adsorption position of individual cytosine molecules on $\mathrm{CaF}_{2}(111)$. Here, we employed the SIESTA DFT package ${ }^{39}$ with DZP basis sets, Troullier-Martins pseudopotentials, and a mesh cutoff energy of 100 Ry. Due to the structural rigidity of $\mathrm{CaF}_{2}(111)$, only one F-Ca-F triple layer was used to simulate the substrate. One molecule of cytosine was placed on a $2 \times 2$ surface unit cell. Full relaxation of all molecular coordinates results in the optimum structure as shown in Fig. 4(a) associated with an adsorption binding energy of $0.65 \mathrm{eV}$. In this configuration, the negatively charged part of the molecule around the oxygen atom is located above a calcium ion of the second layer while the positive part of the molecule around the amine group is above two fluorine ions of the topmost surface layer, pointing to the electrostatic nature of the molecule-substrate interaction. To investigate mobility along the surface, the molecule was shifted laterally and rotated around the surface normal (all controlled by two position and one rotation parameter), while allowing all other molecular coordinates to relax. These calculations reveal that diffusion is possible in all directions associated with barriers of about $0.5 \mathrm{eV}$. All energies resulting from DFT in this system should be taken with some care. On the one hand, the use of only one F-Ca-F triple layer might be considered too thin; however, test calculations with thicker slabs demonstrate convergence of the single-layer calculations. On the other hand, relaxations of the substrate are neglected. The most severe approximation, however, lies in the use of the DFT itself for a weakly bonded adsorbate system, leading to uncertainties (probably underestimation) in the adsorption energy. Based on more elaborate correlation-energy studies for other systems ${ }^{40}$ we estimate that the adsorption energy might be in the range of $0.6-1.0 \mathrm{eV}$. Also, we estimate the uncertainty of our diffusion energies to be $\geq 0.1 \mathrm{eV}$. Note that, in spite of its simplicity, the local-density approximation to DFT employed in this paper has been shown to be the most reliable density functional for weakly interacting systems. ${ }^{40}$ Based on the optimum adsorption position obtained by DFT, we develop a model of the trimer structure as shown in Fig. 4(b). In this configuration, the molecules are placed at the optimum adsorption position as shown in Fig. 4(a). As can be seen, the molecules can readily form a threemembered ring by hydrogen bonding between the oxygen atoms and the hydrogen atoms of the amino group. The resulting distance between the nitrogen and the oxygen atoms is about $3 \AA$ in the unrelaxed configuration. It is, however, very likely that the molecules rotate slightly inwards around an axis through the oxygen atom normal to the surface in order to reduce the nitrogen-oxygen distance thereby increasing the hydrogen bond strength. The cost of a $20^{\circ}$ rotation is about $0.3 \mathrm{eV}$ per molecule as obtained from the DFT calculation while the gain for forming a strong hydrogen bond can be as high as $0.65 \mathrm{eV}^{41} \mathrm{We}$ want to stress that the resulting arrangement implies a well-defined orientation with threefold symmetry of the trimer on the surface. This orientation is exactly the one observed experimentally.

Note that the molecular arrangement is prochiral, i.e., the molecules themselves are nonchiral, while two mirrorsymmetric enantiomers form when constraint to a twodimensional surface. As a consequence of this surface chirality, also the trimers exist in two enantiomers and both of them should be present on the surface. Based on our NCAFM images, it is, however, not possible to differentiate between the two forms.

We now discuss a possible structure model for the tetramers. A very likely explanation for tetramers is that they consist of a trimer with an additional cytosine molecule attached to the trimer ring as shown in the model in Fig. 4(c). When assuming the same adsorption position with respect to the $\mathrm{CaF}_{2}$ (111) lattice, the molecule might anchor to the trimer by electrostatic interaction between the negatively charged part around the oxygen atom of the additional molecule and the positively charged part of one of the trimer molecules. Assuming such a molecular structure, one anchoring site exists, which should appear in three different orientations due to the threefold symmetry of the substrate. Indeed, we observe (i) the correct anchoring position with respect to the trimer ring and (ii) the three equivalent tetramer configurations in roughly equal amounts in our experiments. Two out of these three configurations are found in Fig. 3(a), (2) and (3). ${ }^{42}$ 
In the following, we discuss the diffusion energies involved in the formation of the observed trimer structures. We never observed mobile individual molecules nor hopping events of individual molecules. The smallest stable entity observed are clusters of three molecules. This result readily indicates that individual molecules are mobile at room temperature. To compare this experimental result with the calculated diffusion barrier of $\approx 0.5 \mathrm{eV}$, we consider the hopping rate equation ${ }^{43,44}$ and calculate the hopping rate corresponding to an energy barrier of $0.5 \mathrm{eV}$. The attempt frequency $h_{0}$ of organic molecules on metallic surfaces is known to be on the order of $10^{10}-10^{13} \mathrm{~s}^{-1} \cdot{ }^{43,45}$ Using this range for the attempt frequency results in a hopping frequency of 40 to 40000 events per second, thus, individual molecules are highly mobile at room temperature, in good agreement with our experimental findings. On the other hand, when assembled into a three-membered ring, the cytosine trimers are observed to be immobile at room temperature. When comparing the diffusion barrier of a cluster with those of the individual molecules, it is important to consider whether the molecules within the cluster maintain their optimum adsorption position or not. As long as the optimum adsorption position is preserved, a simple summation of the individual diffusion barriers can serve as a rough estimate for the diffusion barrier of the cluster. As we see from comparing our DFT calculations with the experimental results, the position of the cytosine molecules within the clusters is very close to the optimum adsorption position of an individual molecule. Thus, in this case, the summation of diffusion barriers is justified, yielding a diffusion barrier of $\approx 1.5 \mathrm{eV}$ for a trimer, which is far too high to be overcome at room temperature. Thus, the relatively large diffusion barrier of individual molecules in the first place in combination with the ability to assemble in hydrogen-bonded rings results in the formation of stable molecular structures on the terraces at room temperature.

In conclusion, our results indicate that choosing a welladapted molecule-substrate system allows controlled structure formation on an insulating surface. Exploiting the cooperative mechanism between the polar molecule-substrate interaction and the molecule-molecule hydrogen bonds appears to be responsible for creating trimers on terraces away from step edges or other nucleation centers. This approach should be applicable for other molecule/substrate systems, extending controlled molecular self-assembly from metallic surfaces to dielectric substrates.

\section{ACKNOWLEDGMENTS}

This work has been supported by the German Research Foundation (DFG) through the Emmy Noether-program and the Niedersachsen $\mathrm{PhD}$ program "Synthesis and Characterisation of Surfaces and Interfaces assembled with Clusters and Molecules".
*Present address: Institut für Physikalische Chemie, Johannes Gutenberg-Universität Mainz, Jakob-Welder-Weg 11, 55099 Mainz, Germany.

${ }^{\dagger}$ Present address: Interdisciplinary Nanoscience Center (iNANO) and Department of Physics and Astronomy, University of Aarhus, Ny Munkegade, DK-8000 Aarhus C, Denmark.

*kuehnle@uni-mainz.de

${ }^{1}$ C. Joachim, J. K. Gimzewski, and A. Aviram Nature (London) 408, 541 (2000).

${ }^{2}$ M. Böhringer, K. Morgenstern, W.-D. Schneider, R. Berndt, F. Mauri, A. De Vita, and R. Car, Phys. Rev. Lett. 83, 324 (1999).

${ }^{3}$ T. Yokoyama, S. Yokoyama, T. Kamikado, Y. Okuno, and S. Mashiko, Nature (London) 413, 619 (2001).

${ }^{4}$ A. Kühnle, L. M. Molina, T. R. Linderoth, B. Hammer, and F. Besenbacher, Phys. Rev. Lett. 93, 086101 (2004).

${ }^{5}$ J. A. Theobald, N. S. Oxtoby, M. A. Phillips, N. R. Champness, and P. H. Beton, Nature (London) 424, 1029 (2003).

${ }^{6}$ J. V. Barth, Annu. Rev. Phys. Chem. 58, 375 (2007).

${ }^{7}$ A. Kühnle, Curr. Opin. Colloid Interface Sci. 14, 157 (2009).

${ }^{8}$ S. A. Burke, J. M. Mativetsky, R. Hoffmann, and P. Grütter, Phys. Rev. Lett. 94, 096102 (2005).

${ }^{9}$ S. A. Burke, J. M. Mativetsky, S. Fostner, and P. Grütter, Phys. Rev. B 76, 035419 (2007).

${ }^{10}$ S. A. Burke, W. Ji, J. M. Mativetsky, J. M. Topple, S. Fostner, H. J. Gao, H. Guo, and P. Grütter, Phys. Rev. Lett. 100, 186104 (2008).

${ }^{11}$ U. Zerweck, C. Loppacher, and L. M. Eng, Nanotechnology 17, S107 (2006).
${ }^{12}$ Ch. Loppacher, U. Zerweck, L. M. Eng, S. Gemming, G. Seifert, C. Olbrich, K. Morawetz, and M. Schreiber, Nanotechnology 17, 1568 (2006).

${ }^{13}$ Ch. Loppacher, U. Zerweck, D. Koehler, M. Rodenstein, E. Jaehne, R. Luther, H. J. Adler, and L. M. Eng, Nanotechnology 18, 084003 (2007).

${ }^{14}$ Th. Dienel, C. Loppacher, S. C. B. Mannsfeld, R. Forker, and T. Fritz, Adv. Mater. (Weinheim, Ger.) 20, 959 (2008).

${ }^{15}$ T. Kunstmann, A. Schlarb, M. Fendrich, T. Wagner, R. Möller, and R. Hoffmann, Phys. Rev. B 71, 121403(R) (2005).

${ }^{16}$ M. Fendrich and T. Kunstmann, Appl. Phys. Lett. 91, 023101 (2007).

${ }^{17}$ Th. Glatzel, L. Zimmerli, S. Koch, S. Kawai, and E. Meyer, Appl. Phys. Lett. 94, 063303 (2009).

${ }^{18}$ S. Maier, L.-A. Fendt, L. Zimmerli, T. Glatzel, O. Pfeiffer, F. Diederich, and E. Meyer, Small 4, 1115 (2008).

${ }^{19}$ L. Nony, R. Bennewitz, O. Pfeiffer, E. Gnecco, A. Baratoff, E. Meyer, T. Eguchi, A. Gourdon, and C. Joachim, Nanotechnology 15, S91 (2004).

${ }^{20}$ L. Nony, E. Gnecco, A. Baratoff, A. Alkauskas, R. Bennewitz, O. Pfeiffer, S. Maier, A. Wetzel, E. Meyer, and C. Gerber, Nano Lett. 4, 2185 (2004).

${ }^{21}$ J. M. Mativetsky, S. A. Burke, S. Fostner, and P. Grütter, Nanotechnology 18, 105303 (2007).

${ }^{22}$ J. M. Mativetsky, Y. Miyahara, S. Fostner, S. A. Burke, and P. Grütter, Appl. Phys. Lett. 88, 233121 (2006).

${ }^{23}$ H.-P. Weber and B. M. Craven, Acta Crystallogr., Sect. B: Struct. Sci. 46, 532 (1990). 
${ }^{24}$ K. M. Guckian, B. A. Schweitzer, R. X.-F. Ren, C. J. Sheils, D. C. Tahmassebi, and E. T. Kool, J. Am. Chem. Soc. 122, 2213 (2000).

${ }^{25}$ P. U. Civcir, J. Phys. Org. Chem. 14, 171 (2001).

${ }^{26}$ M. A. Spackman, P. Munshi, and B. Dittrich, ChemPhysChem 8, 2051 (2007).

${ }^{27}$ C. T. Campos and F. E. Jorge, Int. J. Quantum Chem. 109, 285 (2009).

${ }^{28}$ R. Otero, M. Lukas, R. E. A. Kelly, W. Xu, E. Lægsgaard, I. Stensgaard, L. N. Kantorovich, and F. Besenbacher, Science 319, 312 (2008).

${ }^{29}$ T. R. Albrecht, P. Grütter, D. Horne, and D. Rugar, J. Appl. Phys. 69, 668 (1991).

${ }^{30}$ J. Schütte, R. Bechstein, P. Rahe, M. Rohlfing, A. Kühnle, and H. Langhals, Phys. Rev. B 79, 045428 (2009).

${ }^{31}$ S. Gritschneder, Y. Namai, Y. Iwasawa, and M. Reichling, Nanotechnology 16, S41 (2005).

${ }^{32}$ P. Rahe, R. Bechstein, J. Schütte, F. Ostendorf, and A. Kühnle, Phys. Rev. B 77, 195410 (2008).

${ }^{33}$ L. Tröger, J. Schütte, F. Ostendorf, A. Kühnle, and M. Reichling, Rev. Sci. Instrum. 80, 063703 (2009).

${ }^{34}$ M. Reichling and C. Barth, Phys. Rev. Lett. 83, 768 (1999).

${ }^{35}$ A. S. Foster, C. Barth, A. L. Shluger, and M. Reichling, Phys.
Rev. Lett. 86, 2373 (2001)

${ }^{36}$ C. Barth, A. S. Foster, M. Reichling, and A. L. Shluger, J. Phys.: Condens. Matter 13, 2061 (2001).

${ }^{37}$ A. S. Foster, C. Barth, A. L. Shluger, R. M. Nieminen, and M. Reichling, Phys. Rev. B 66, 235417 (2002).

${ }^{38}$ M. L. Sushko, A. Y. Gal, M. Watkins, and A. L. Shluger, Nanotechnology 17, 2062 (2006).

${ }^{39}$ J. M. Soler, E. Artacho, J. D. Gale, A. García, J. Junquera, P. Ordejon, and D. Sanchez-Portal, J. Phys.: Condens. Matter 14, 2745 (2002).

${ }^{40}$ M. Rohlfing and T. Bredow, Phys. Rev. Lett. 101, 266106 (2008).

${ }^{41} \mathrm{G}$. A. Jeffrey, An Introduction to Hydrogen Bonding, Topics in Physical Chemistry (Oxford University Press, New York, 1997).

${ }^{42} \mathrm{We}$ want to note that the existence of the tetramer structures serves as an experimental proof demonstrating that the trimer structures are not just due to imaging with a triple tip, as tetramers would not be observable with a triple tip.

${ }^{43}$ J. V. Barth, Surf. Sci. Rep. 40, 75 (2000).

${ }^{44}$ G. L. Kellogg, Surf. Sci. Rep. 21, 1 (1994).

${ }^{45}$ J. Weckesser, J. V. Barth, and K. Kern, J. Chem. Phys. 110, 5351 (1999). 